\title{
Asthma drug ratios and exacerbations: claims data from universal health coverage systems
}

\author{
Laurent Laforest ${ }^{1}$, Idlir Licaj', Gilles Devouassoux², Gérard Chatte³ \\ Jennifer Martin ${ }^{1}$ and Eric Van Ganse ${ }^{1,2}$
}

Affiliations: 'Unité de Pharmacoépidémiologie, CHU-Lyon, Faculté d'Odontologie, UMR 5558-LBBE, CNRS, Université Claude Bernard, Lyon, ${ }^{2}$ Service de Pneumologie, Hôpital de la Croix Rousse, Hospices Civils de Lyon, Lyon, and ${ }^{3}$ Liberal Chest Physician, Caluire, France.

Correspondence: E. Van Ganse, Unité de Pharmacoépidémiologie, 11, rue Guillaume Paradin, 69372 Lyon, Cedex 08, France. E-mail: eric.van-ganseduniv-lyon1.fr

ABSTRACT In claims data, controller-to-total asthma drug ratios may reflect adequacy of disease management. We verified whether asthma patients with high ratios $(\geqslant 50 \%)$ experienced fewer asthmarelated outcomes. Two ratios were studied: that of the inhaled corticosteroids to total asthma drug (ICS/ R03) and that of the inhaled corticosteroids plus leukotriene antagonist receptors-to-total asthma drug (ICS+LTRA/R03).

Patients aged 13-40 years, with $\geqslant 3$ respiratory drugs dispensed prescriptions in 2005 were selected from the French national claims data. After excluding null ratios, two groups were defined according to ratio values in 2007: low-ratio group $(0 \%<$ ratio $<50 \%)$ and high-ratio group (ratio $\geqslant 50 \%$ ). For both ratios, asthma-related outcomes and medical-resource utilisation were compared between groups.

Of 2162 patients (mean age 27 years and 52\% female), patients with non-null ratios were $81 \%$ and $85 \%$ for ICS/R03 and ICS+LTRA/R03 ratios, respectively. Patients with high ratios were less likely to receive oral corticosteroids than those in the low-ratio group (relative risk $0.79,95 \%$ CI $0.72-0.88$, and $0.80,95 \%$ CI 0.72-0.88, for ICS/R03 and ICS+LTRA/R03, respectively). High ratio groups also presented fewer asthmarelated hospitalisations. Significant negative correlations were also observed for both ratios, when studied quantitatively, according to patients' dispensed level of oral corticosteroids in 2007.

In claims data, both ICS/R03 and ICS+LTRA/R03 $\geqslant 50 \%$ were related to fewer asthma-related outcomes. Ratios should be explored to identify asthma patients at risk of exacerbations. Low ratios can be considered as risk factors of exacerbation whatever the underlying cause.

@ERSpublications

Asthma patients with therapeutic ratios $\geqslant 50 \%$ had fewer asthma exacerbation markers, suggesting better control http://ow.ly/tHa59

Received: June 132013 | Accepted after revision: Nov 182013

Support statement: This study was supported by a grant from the French National Health Insurance Agency (grant number 11/6/2009/CT/49).

Conflict of interest: Disclosures can be found alongside the online version of this article at www.erj.ersjournals.com

Copyright @ERS 2014 


\section{Introduction}

Regular use of controller medications remains a key issue in asthma disease management. Observational studies have shown that inconsistent use of controller medications has a direct impact on medical resource utilisation $[1,2]$. One promising approach to identifying patients with inconsistent controller use is through claims data, which include exhaustive information on reimbursed medical resource utilisation in the insured population. Using these data to identify patients at risk of insufficient control of chronic diseases, such as asthma, may help improve disease management [3].

Authors have investigated ratios measuring the proportion of dispensed controllers in total asthma therapy as a marker of the quality of care $[4,5]$. Studies using these ratios have consistently shown higher levels of asthma-related hospital admissions and emergency room visits for patients with lower ratios [6]. The impact of ratios on oral corticosteroids (OCSs) has been less extensively explored.

Furthermore, unlike controller-to-total asthma drug ratio, the inhaled corticosteroid (ICS)-to-total asthma drug ratio (ICS/R03, where R03 codes for asthma therapy according to the Anatomical Therapeutic and Chemical (ATC) classification system) has been poorly studied. It is also unclear whether considering only ICSs or both ICS and leukotriene receptor antagonist (LTRA) agents in the numerator ratio yield concordant results.

Lastly, previous studies have relied on data from health management organisations, on specific populations and typically salaried persons with specific processes of care, and there is a need to replicate such studies in other healthcare systems with universal coverage, including patients with a high level of deprivation.

We investigated whether low ICS/R03 ratios, which suggest less consistent ICS exposure for a given disease severity, were related to more frequent asthma-related exacerbations and greater overall medical resource utilisation. Parallel investigations were conducted for ICS+LTRA/R03 ratio to verify whether both ratios yielded concordant findings. For each ratio, we also looked for differences in patient characteristics and controller therapy between the high and low ratio groups.

\section{Methods}

Study design and timelines

A historical cohort (2005-2008) was obtained from the Permanent Sample of Health Insurance Beneficiaries (EGB), a 1/97th random sample of the French National Claims Data Beneficiaries with individual linkage between ambulatory and hospital care. We selected patients aged 13-40 years on January 1, 2005, with continuous follow-up between 2005 and 2008, and $\geqslant 3$ asthma drugs (R03 code) dispensed in three different quarters during 2005. The year 2006 was used to assess patients' asthma severity. Severity was approached by the total number of asthma drug classes (including OCSs) dispensed in 2006. We conducted analyses in 2007 and 2008 (fig. 1). This observational study was conducted on anonymised claims data, and the National Informatics and Liberty Committee has delivered an overall authorisation to use EGB data for research purposes.

\section{Data collected}

Patient characteristics were age, sex, long-term disease status and free-access-to-care status. Long-term disease status allows severe patients to receive therapy without advancing payment in pharmacies. Freeaccess-to-care status enables patients with socioeconomic difficulties to receive free medical care. Reimbursed therapy included asthma medications, OCSs and antibiotics. Medical contacts (family physicians and respiratory physicians) were counted in the database. We identified hospitalisations with asthma as primary or secondary diagnosis. The primary diagnosis corresponds to the disease that incurred the majority of the resources used during a given hospital stay.

\section{ICS/R03 and ICS+LTRA/R03 ratios}

The ratio of the number of ICS units (whether in fixed combination with long-acting $\beta$-agonists (LABAs) or not) to the overall units of asthma drugs (R03 ATC classification) dispensed in 2007 was computed. Asthma drug therapy included ICSs, LABAs, LABA-ICS fixed combinations, short-acting $\beta$-agonists (SABAs), LTRAs, anticholinergics, anticholinergics-SABA fixed combinations, xanthines and cromones (R03 according to ATC classification). Based on this ICS/R03 ratio in 2007, three groups were defined: $0 \%$ (no ICS), $0 \%<$ ratio $<50 \%$ (low ratio) and ratio $\geqslant 50 \%$ (high ratio). The choice of the $50 \%$ threshold was based on previous studies using controller-to-total respiratory drug ratios [4]. The ratio of ICS plus LTRA to total asthma drug (ICS+LTRA/R03), including both LTRA and ICS units in the numerator, was also computed and studied. 


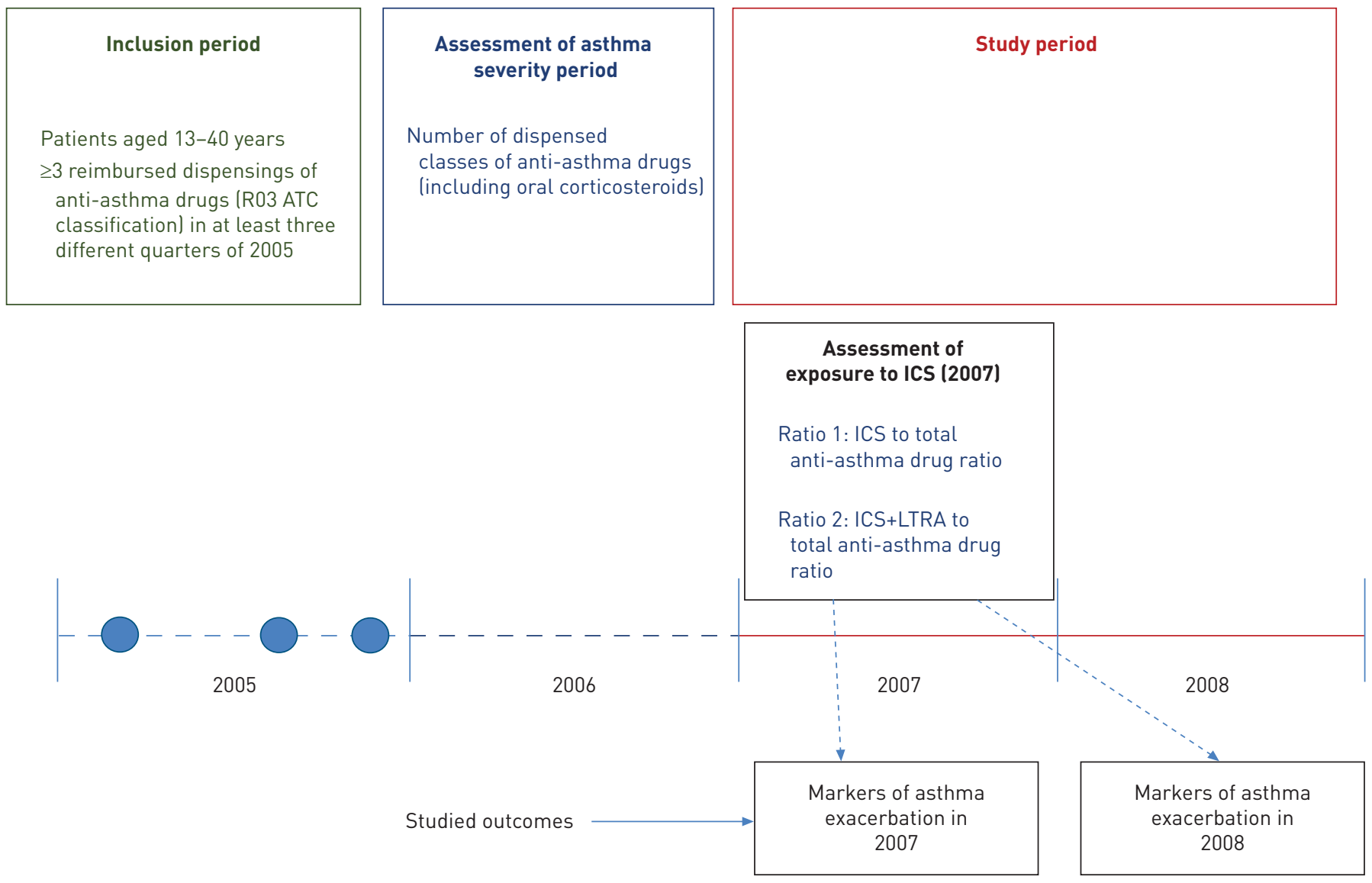

FIGURE 1 Study design. ICS: inhaled corticosteroid; R03 ATC: R03 coding for asthma therapy according to the Anatomical Therapeutic and Chemical classification system; LTRA: leukotriene receptor antagonist.

\section{Outcome criteria}

Outcomes were proxies for asthma exacerbations in 2007 and 2008 [7]. The main outcomes were dispensing of the drug classes commonly used to treat asthma exacerbations, i.e. OCSs (percentage of patients with at least one drug dispensed and mean number of prescriptions dispensed). The annual number of visits to a family physician was also investigated.

Other criteria were hospitalisations for asthma as primary diagnosis and hospitalisations for asthma as primary or secondary diagnosis. Percentages of patients with at least one hospitalisation, the number of stays per patient and the hospitalisation-related costs were studied in 2007 and 2008. Levels of SABAs dispensed were not used as an outcome, as they were included in the denominator of ICS/R03 ratios.

\section{Analyses}

Analyses were conducted in parallel for ICS/R03 and ICS+LTRA/R03 ratios. For both ratios, all analyses were restricted to low- and high-ratio groups (no null-ratio values).

First, baseline characteristics and controller therapy dispensed in 2007 were compared between groups. The outcomes were compared between groups in 2007 and in 2008 using standard Chi-squared test (Fisher's exact test when appropriate), ANOVA or Wilcoxon-Kruskal-Wallis test.

Then, multivariate Poisson models were run to compute the risks of receiving OCSs in 2007 (at least one dispensed prescription) for both ratios. It was verified whether patients in the high-ratio group presented lower risks of receiving OCSs in 2007 compared with those in the low-ratio group. Models were adjusted for age, sex, long-term disease status, free-access-to-care status, dispensing of at least one LABA-ICS fixed combination in 2007, and ratio group in 2007 (high versus low). Complementary models were also computed with an additional adjustment for the baseline severity variable. The aim was to verify the extent to which differences in outcomes between groups were due to factors other than baseline severity. In the absence of clinical and spirometric data, adjustment for baseline severity was approached by the number of 
dispensed respiratory drug classes in 2006, including OCSs $(0-1,2-3$ and $>3$ ) [8]. Similar analyses were also conducted for receiving OCSs in 2008.

Owing to their low frequency, asthma-related hospitalisations were not studied in multivariate analyses. All analyses were performed on SAS software version 9.3 (SAS Institute Inc., Cary, NC, USA).

\section{Additional analyses with quantitative ratios}

Additional analyses with quantitative ratios were performed for ICS/R03 and ICS+LTRA/R03 ratios. In 2007 , both ratios were studied according to the number of dispensed units of OCS in 2007, and then in 2008. Univariate and multivariate Poisson regressions were conducted. Multivariate analyses were adjusted for the same cofactors as those in the main analyses.

\section{Results}

\section{Descriptive results}

Both ratios were computed for 2162 patients (mean age 27 years and 52\% female) who met the inclusion criteria, with at least one asthma drug dispensed in 2007. The proportions of patients in the no-ICS (null ratio), and low- and high-ICS ratio groups were $19 \%, 36 \%$ and $45 \%$, respectively. Respective percentages for ICS+LTRA/R03 ratios were 15\%, 25\% and 59\%. After excluding null ratios, analyses were conducted in 1758 and 1827 patients for ICS/R03 and ICS+LTRA/R03 ratios, respectively.

\section{Patient characteristics and dispensed controller therapy according to the ICS/R03 and ICS+LTRA/ R03 ratios}

For both ratios, patients in the low-ratio groups more frequently had free-access-to-care and long-termdisease status (table 1). Conversely, there were no significant differences by either sex or age. Compared with the low-ratio groups, patients in the high-ratio groups received significantly more ICS units, particularly as LABA-ICS fixed combinations; received fewer LABAs (not combined); and had fewer primary care consultations in 2007, while they tended to visit more respiratory physicians, although this was not significant (table 1). Patients in the ICS/R03 high-ratio group received fewer LTRAs than those in the ICS/R03 low-ratio group. This was reversed for the ICS+LTRA/R03 ratio.

\section{Asthma-related outcomes according to ICS/R03 and ICS+LTRA/R03 ratios}

Compared with the low-ratio groups, patients in the high-ratio groups had fewer dispensed prescriptions of OCSs in 2007 and in 2008 (table 2). They also experienced fewer asthma-related hospitalisations.

\section{Multivariate analyses}

Patients with a high ratio had a significantly lower risk of receiving OCSs in 2007 compared with those in the low-ratio group. For both ratios, the association persisted in the complementary models, even after adjustment for baseline severity. Similar trends, although less marked, were also observed for receiving OCSs in 2008 (table 3).

\section{Additional analyses with quantitative ratios}

Significant decreases in mean ratios were observed with the number of dispensed units of OCSs in 2007 for both ICS/R03 and ICS+LTRA/R03 ratios. Similar downward trends, although less marked, also appeared with the OCSs dispensed in 2008. Results of regression models of ratios with OCS dispensing in 2007 remained significant in multivariate analyses (table 4).

\section{Discussion}

In our sample of 2162 patients, ICS/R03 and ICS+LTRA/R03 ratios were non-null ratios in $81 \%$ and $85 \%$, respectively. In those with non-null ratios, the ICS/R03 high-ratio group accounted for $55 \%$ of patients. It accounted for $70 \%$ of the ICS+LTRA/R03 ratio. Overall, patients in the low-ratio groups experienced more use of OCSs and more asthma-related hospitalisations than those in the high-ratio group (table 2).

For both ratios, the risk of receiving OCSs in 2007 was significantly lower in the high-ratio group compared with the low-ratio group, even after adjustment for asthma severity (table 3). The complementary models indicate that the potential difference in asthma severity between groups can explain only in part the lower risks of outcomes in the high ratio group. Likewise, patients with a low ratio experienced more asthmarelated hospitalisations.

Additional analyses showed a limited downward variation of quantitative ratios with patients' level of dispensed OCSs, although results were statistically significant in univariate analyses (table 4). 
TABLE 1 Patients' characteristics according to ICS/R03 drug ratio in 2007

ICS/R03

ICS+LTRA/R03

\begin{tabular}{cc}
\hline $\begin{array}{c}\text { Low-ratio } \\
\text { group }\end{array}$ & $\begin{array}{c}\text { High-ratio } \\
\text { group }\end{array}$
\end{tabular}

$\begin{array}{ccc}\begin{array}{c}\text { Low-ratio } \\ \text { group }\end{array} & \begin{array}{c}\text { High-ratio } \\ \text { group }^{5}\end{array} & \text { p-value }\end{array}$

\section{Subjects $\mathbf{n}$}

Mean age years $^{\prime}$

Males \%

Long-term disease status $\#$

Free-access-to-care status ${ }^{\text {Tा }} \%$

Medical resource utilisation in 2007

$\geqslant 1$ visit to a respiratory physician ${ }^{++} \%$

Mean visits to family physician

\section{Controllers}

ICS lother than LABA-ICS fixed combination)

$\geqslant 1$ unit $\%$

Mean number of dispensed units

LABA-ICS fixed combination

$\geqslant 1$ unit $\%$

Mean number of dispensed units

Mean number of ICS units (any type)

LABA (not in LABA-ICS fixed combination) $\geqslant 1$ unit \%

Mean number of dispensed units

LTRA

$\geqslant 1$ unit $\%$

Mean number of dispensed units

$\begin{array}{lr}792 & 966 \\ 27.2 & 28.1 \\ 45.4 & 47.7 \\ 12.6 & 7.4 \\ 18.3 & 13.8 \\ & \\ 2.9 & 4.4 \\ 7.0 & 5.7\end{array}$

\section{4}

2.02

65.5

3.04

5.06

\section{7}

28.7

1.91

45.3

2.77

966

28.1

47.7

7.4

13.8

4.4

5.7

\section{0}

0.98

4.79

5.77

0.04
0.34
0.0003
0.009

0.09
0.0002

$<0.0001$

$<0.0001$

$<0.0001$

$<0.0001$

6.6

0.23

$<0.0001$

$<0.0001$

11.6

0.39

$<0.0001$

$<0.0001$

\section{8}

27.7

44.0

11.7

18.8

2.6

7.3

53.1

2.12

58.2

2.40

4.52

33.3

2.30

21.0

0.90
1279

27.4

48.2

8.7

14.4

4.2

5.7

$<0.0001$

ICS: inhaled corticosteroid; R03: Anatomical Therapeutic and Chemical classification system code for asthma therapy; LTRA: leukotriene receptor antagonists. ${ }^{\#}$ : $0 \%<\mathrm{ICS} / \mathrm{R03}<50 \%$; ${ }^{\varphi}$ : ICS/R03 $\geqslant 50 \% ;{ }^{+}: 0 \%<\mathrm{ICS}+\mathrm{LTRA} / \mathrm{RO} 3<50 \%$; ${ }^{\S}$ : ICS+LTRA/R03 $\geqslant 50 \%$; ${ }^{f}$ : age in $2005 ;{ }^{\# \#}$ : patients are

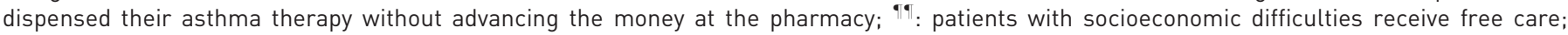
${ }^{++}$: respiratory physicians with a private consulting room (dispensing resulting from prescriptions from hospital respiratory physicians cannot be identified in the datal.

Overall, our main findings are in line with previous studies that reported fewer asthma-related hospitalisations and emergency room visits when the controller-to-total asthma drug ratio was $\geqslant 50 \%[4$, 5]. A lower risk of belonging to the high ratio group was found in controller-treated patients with at least two refills of OCSs (OR=0.89, 95\% CI 0.82-0.98) [9]. However, SCHATz et al. [10] identified a significantly lower risk of receiving OCSs only at a $90 \%$ threshold. The exclusion of null -ratios and potential differences in prescribing habits between countries may account for these differences.

Indeed, better asthma control and quality of life have been found by SCHATZ et al. [11] in patients with a controller-to-total asthma drug ratio of $\geqslant 50 \%$. In addition to lower exacerbation-related medical resource utilisation during the same calendar year, a high ICS/R03 ratio seems to predict outcomes in the following year, reinforcing a potential role for this marker. However, caution is needed given the more limited differences observed between groups with outcomes measured in 2008 (table 2).

Other differences between high- and low-ratio users are noteworthy. The number of medical visits was higher in patients with low ratios, possibly due to more frequent unscheduled visits. As expected, dispensed levels of ICS were higher in the high-ratio groups, although these differences between low- and high-ratio groups were not large (table 1). In addition, patients with high ratios tended to receive more LABA-ICS fixed combinations (table 1). This finding has also been observed by Broder et al. [9]. Patients under LABA-ICS fixed combinations are less likely to require SABAs and do not use LABAs, which would decrease the ratio denominator. More regular dispensing of LABA-ICS fixed combinations compared with ICS alone has also been observed in claims data [12-14]. However, multivariate models clearly indicated that patients in the high-ratio groups were less likely to receive OCSs, irrespective of concomitant use of LABA-ICS fixed combinations.

The greater frequency of patients visiting specialists in the high-ratio group is in line with previous studies [9]. A better quality of care provided by respiratory physicians could account for a higher ICS dispensation. 


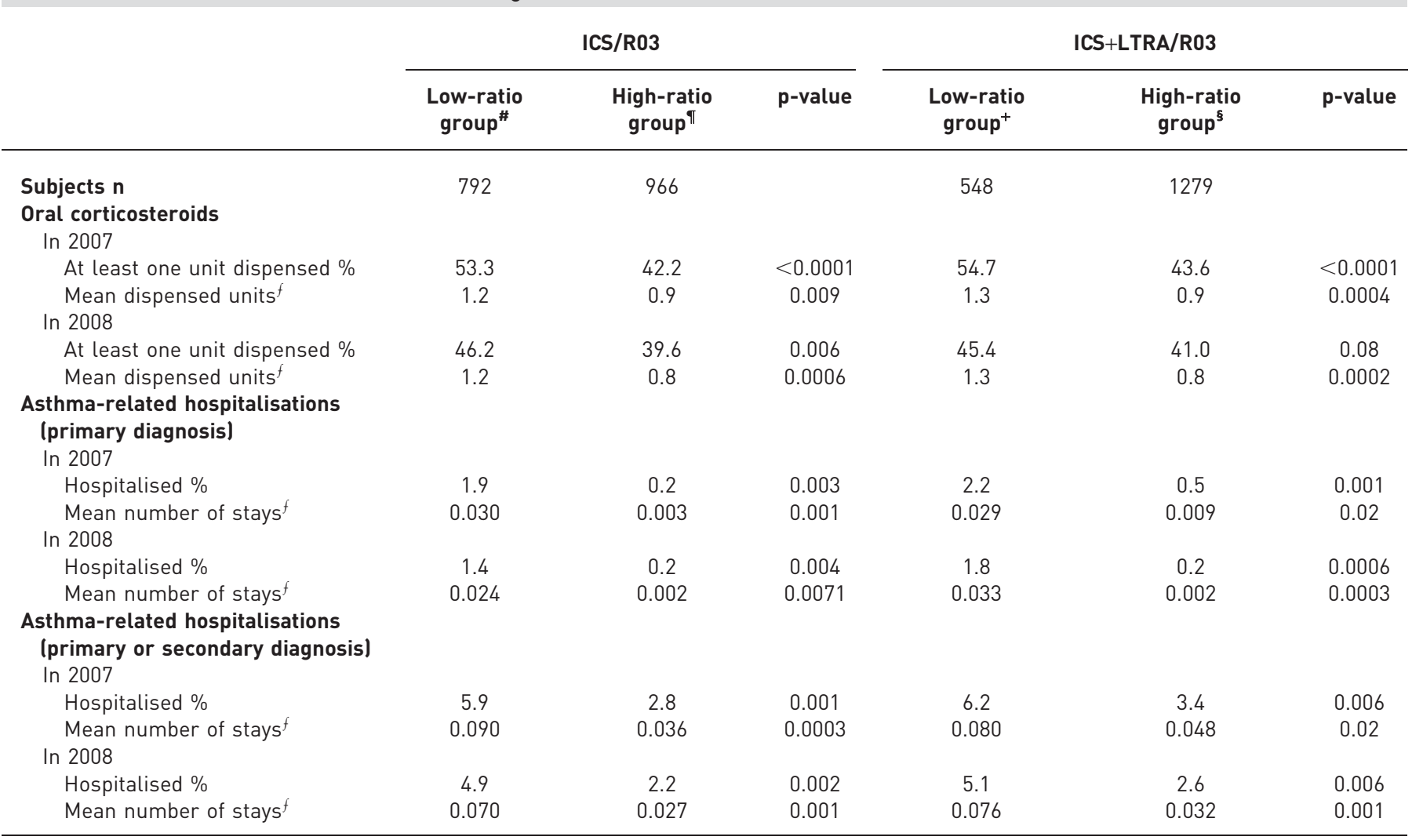

ICS: inhaled corticosteroid; R03: Anatomical Therapeutic and Chemical classification system code for asthma therapy; LTRA: leukotriene receptor

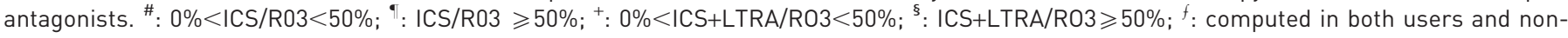
users.

Nonetheless, caution is required, given the low number of private-sector respiratory physicians in our data and nonsignificant results.

Patients with free-access-to-care status were more common in low-ratio groups, suggesting that social difficulties may be an obstacle to reaching high ratios. Patients with long-term disease status were also more common among low-ratio users, suggesting potentially more severe asthma in this group with high levels of medical resource utilisation. These points require confirmation in new studies.

The ICS/R03 and ICS+LTRA/R03 ratio groups differed as to distribution, using the 50\% cut-off value. In particular, patients using both ICS and LTRA were more likely to belong to the ICS/R03 low-ratio group, which was not the case for the ICS+LTRA/R03 ratio. Expectedly, the ICS+LTRA/R03 high-ratio group tended to receive more LTRA than the low-ratio group, while it was the other way round for the ICS/R03 ratio (table 1). Further investigations are needed to better understand the differences between both ratios. Our ICS+LTRA/R03 ratio was close to the defintion of SCHATZ et al. [4], although not identical. For instance, cromones and xanthines were not included in the numerator, while the denominator comprised all LABAs, whether or not as LABA-ICS fixed combinations. However, cromones and xanthines are used only marginally. Although there were still LABA users (not combined) at the time of the study (table 1), their proportion has noticeably dropped in France in recent years (personal communication; E. Van Ganse, Unité de Pharmacoépidémiologie, CHU-Lyon, Faculté d'Odontologie, Université Claude Bernard, Lyon, France). Nevertheless, despite these differences in definitions, concordant conclusions were obtained with previous studies, indicating a robustness of these markers of quality of care.

Besides simplicity of computation, an advantage of using the ratios is the consistency of the benefits observed in the high-ratio groups $(\geqslant 50 \%)[6]$, even when the numerator is limited to ICS therapy. The presence of all asthma therapy in the denominator partly accounts for asthma severity, ensuring robustness to an indication bias. 
TABLE 3 Risks of receiving one or more dispensed prescription of OCSs in case of a high ratio value ( $\geqslant 50 \%$ ) for ICS/R03 (models 1 and 2) and for ICS+LTRA/R03 (models 3 and 4) multivariate models

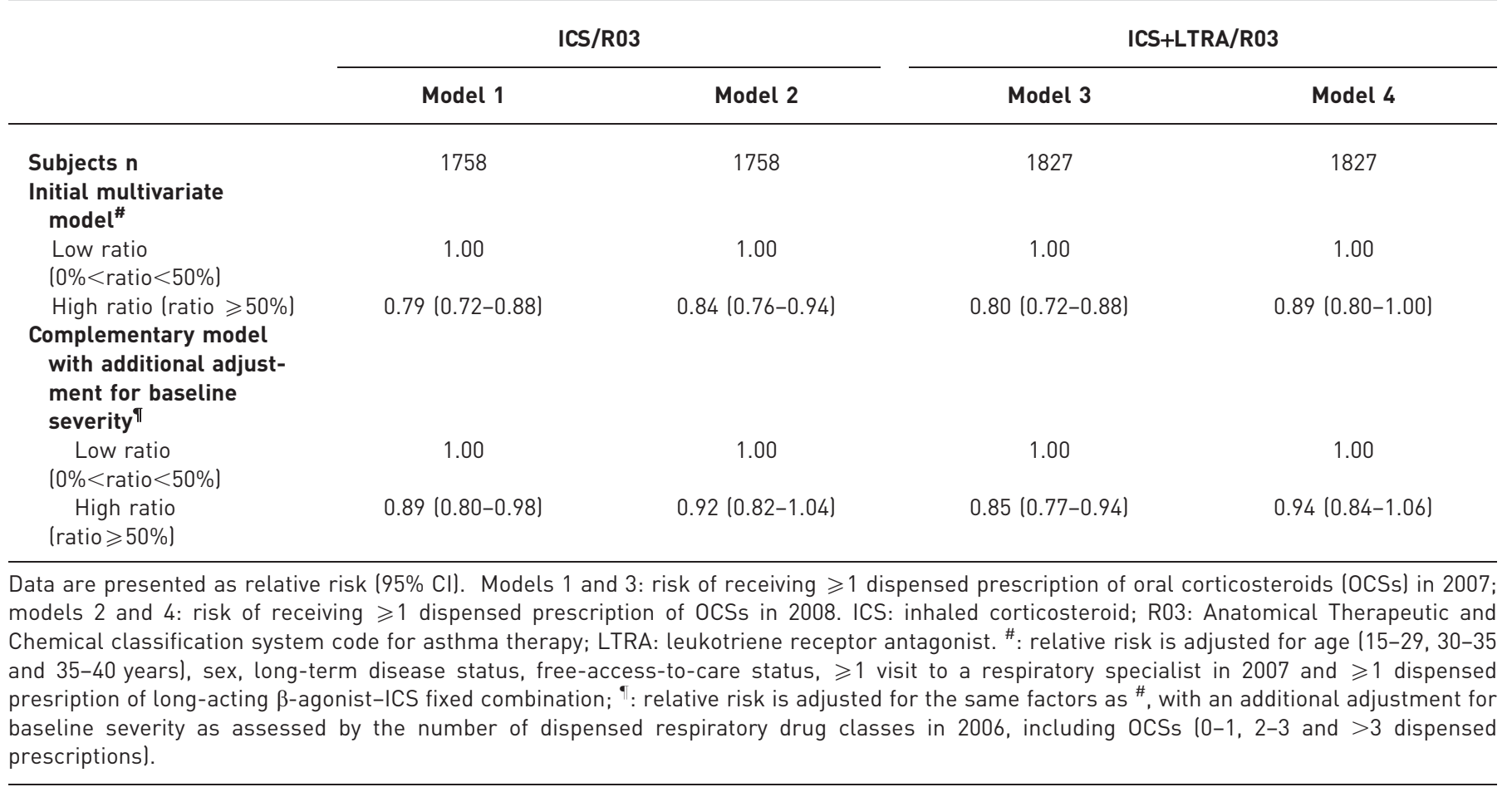

Ratios could be used not only in administrative claims databases to identify asthma patients at risk of exacerbations, but could be of interest for identifying such patients in daily medical practice, e.g. from computerised medical records.

Some limitations must be acknowledged. Hospitalisations with asthma were uncommon, precluding models with these outcomes. It is noteworthy that our specific asthma-related hospitalisation rate was consistent with those previously reported by the National Health Service in the UK [15]. Although OCSs are

TABLE 4 Mean ratio values according to OCS dispensing levels in 2007 and in 2008

\begin{tabular}{cccccc}
\multicolumn{3}{c}{ OCS units dispensed $n$} & & $\begin{array}{c}\text { Univariate Poisson } \\
\text { regression }\end{array}$ & $\begin{array}{c}\text { Multivariate Poisson } \\
\text { regression }^{\#, 9}\end{array}$
\end{tabular}

\begin{tabular}{|c|c|c|c|c|c|c|}
\hline \multicolumn{7}{|c|}{$\begin{array}{l}\text { OCS units dispensed in } 2007 \\
\text { ICS/R03 ratio }\end{array}$} \\
\hline Number & 928 & 428 & 202 & 199 & & \\
\hline Mean \% & 56.1 & 53.1 & 49.9 & 46.5 & $\beta=-0.0055, z=-4.88, p<0.0001$ & $\beta=-0.0026, z=-2.15, p=0.03$ \\
\hline \multicolumn{7}{|c|}{ ICS+LTRA/R03 ratio } \\
\hline Number & 970 & 443 & 209 & 204 & & \\
\hline Mean \% & 64.8 & 61.5 & 59.7 & 56.3 & $\beta=-0.0048, z=-4.45, p<0.0001$ & $\beta=-0.0032, z=-2.86, p=0.0042$ \\
\hline \multicolumn{7}{|c|}{ OCS units dispensed in 2008} \\
\hline \multicolumn{7}{|c|}{ ICS/R03 ratio } \\
\hline Number & 1009 & 388 & 187 & 173 & & \\
\hline Mean \% & 54.9 & 53.3 & 51.8 & 49.0 & $\beta=-0.0033, z=-2.69, p=0.007$ & $\beta=-0.0012, z=-0.89, p=0.37$ \\
\hline \multicolumn{7}{|c|}{ ICS+LTRA/R03 ratio } \\
\hline Number & 1053 & 403 & 195 & 175 & & \\
\hline Mean \% & 63.3 & 63.0 & 61.0 & 57.7 & $\beta=-0.0027, z=-2.32, p=0.02$ & $\beta=-0.0016, z=-1.34, p=0.18$ \\
\hline
\end{tabular}

OCS: oral corticosteroid; ICS: inhaled corticosteroid; R03: Anatomical Therapeutic and Chemical classification system code for asthma therapy; LTRA: leukotriene receptor antagonist. " $:$ z stands for statistical test-value. ": adjusted for age (15-29, 30-35 and 35-40 years), sex, long-term disease status, free-access-to-care status, $\geqslant 1$ visit to a respiratory specialist in 2007, $\geqslant 1$ dispensing of LABA-ICS fixed combination and baseline severity as approached by the number of dispensed respiratory drug classes in 2006, including OCSs (0-1, 2-3 and >3 dispensings). 
recommended in epidemiological studies as outcomes of severe asthma exacerbation [7], they may not be specific for asthma. Additionally, OCSs may be prescribed preventively to patients prior to episodes of exacerbation. However, despite these limitations, significant differences were noted for OCS dispensing levels between ratio groups (tables 3 and 4 ).

Our ratios did not take into account the number of doses per inhaler or the potency of ICS drugs. SCHATZ et al. [10] computed a more elaborate ratio including these variables. However, the basic ratio turned out to be more discriminating for asthma-related outcomes [10]. Severe patients, at higher risk of exacerbations tended to receive more potent ICSs and would more easily qualify as having a higher weighted ratio. Hence, more frequent outcomes would be expected in the high-ratio group when using weighted ratios, which could decrease the difference between low- and high-ratio groups. Additionally, basic ratios are easier to use in practice. Caution is also needed, given the absence of clinical data in claims data.

Also, our sample may not be representative of the overall population of asthma patients as it consists of a subgroup of selected patients with regular follow-up, accounting for 1.3\% of patients aged 13-40 years in the EGB data. Less frequently treated asthma patients, potentially at high risk of adverse outcomes, were not included.

Finally, to define a high ratio, we used the same cut-off $(\geqslant 50 \%)$ as SCHATz et al. [10], as this threshold has yielded consistent conclusions for various outcomes and facilitates comparisons with previous studies. A simple and unique threshold value, such as $50 \%$, is desirable.

Our findings have several implications. Unlike private insurance data, a noticeable advantage of the French claims data lies in their representativeness of the French population, and our data confirm the interest of ratios in non-US healthcare systems. Our findings also support a role for both ICS/R03 and ICS+LTRA/R03 ratios computed from claims data to detect patients potentially at risk of exacerbations. Ratios, independent of the underlying levels of severity/control of the disease and mechanisms, could be used in public health and patient management as a tool to identify patients at higher risk of exacerbation.

Due to the paucity of patient characteristics and clinical data in claims databases, it is difficult to further explain differences between low- and high-ratio groups for medical and personal characteristics and, notably, from a socioeconomic point of view.

Our ability to identify a higher level of OCS dispensing in the case of low ratio using the $50 \%$ threshold does not mean that this value is the most discriminative value for this outcome. The next step of methodological investigations should be the determination of the optimal threshold for OCS dispensing, for both ratios. Another area of research of interest would be the identification of the determinants of such low ratios in a prospective design.

More generally, studying the impact of the ratios with complementary levels of drug exposure (prescriptions, dispensings and actual patient use) could shed light on the reasons for inconsistent use of ICS in asthma management. A low ICS/R03 ratio may be due to irregular dispensings of ICS, e.g. as a result of patients' incomplete adherence to prescribed therapy or irregular prescription of ICSs by physicians [16, 17].

In conclusion, patients with ICS/R03 ratio or ICS+LTRA/R03 ratio $\geqslant 50 \%$ experienced fewer markers of exacerbations, most notably compared with low ICS users, suggesting improved asthma control. Ratios may not only help to identify asthma patients at risk of severe exacerbations from claims data, but they may be of help in clinical practice as a tool to assess the management of asthma.

\section{References}

1 Suissa S, Ernst P, Kezouh A. Regular use of inhaled corticosteroids and the long term prevention of hospitalisation for asthma. Thorax 2002; 57: 880-884.

2 Blais L, Ernst P, Boivin JF, et al. Inhaled corticosteroids and the prevention of readmission to hospital for asthma. Am J Respir Crit Care Med 1998; 158: 126-132.

3 Van Ganse E, van der Linden PD, Leufkens HG, et al. Asthma medications and disease exacerbations: an epidemiological study as a method for asthma surveillance. Eur Respir J 1995; 8: 1856-1860.

4 Schatz M, Nakahiro R, Crawford W, et al. Asthma quality-of-care markers using administrative data. Chest 2005; 128: 1968-1973.

5 Yong PL, Werner RM. Process quality measures and asthma exacerbations in the medicaid population. J Allergy Clin Immunol 2009; 124: 961-966.

6 Schatz M, Zeiger RS. Improving asthma outcomes in large populations. J Allergy Clin Immunol 2011; 128: 273-277.

7 Busse WW, Morgan WJ, Taggart V, et al. Asthma outcomes workshop: overview. J Allergy Clin Immunol 2012; 129: Suppl. 3, S1-S8.

8 Taylor DR, Bateman ED, Boulet LP, et al. A new perspective on concepts of asthma severity and control. Eur Respir J 2008; 32: 545-554.

9 Broder MS, Gutierrez B, Chang E, et al. Ratio of controller to total asthma medications: determinants of the measure. Am J Manag Care 2010; 16: 170-178.

10 Schatz M, Zeiger RS, Yang SJ, et al. Relationship of asthma control to asthma exacerbations using surrogate markers within a managed care database. Am J Manag Care 2010; 16: 327-333. 
11 Schatz M, Zeiger RS, Vollmer WM, et al. The controller-to-total asthma medication ratio is associated with patientcentered as well as utilization outcomes. Chest 2006; 130: 43-50.

12 Stempel DA, Stoloff SW, Carranza Rosenzweig JR, et al. Adherence to asthma controller medication regimens. Respir Med 2005; 99: 1263-1267.

13 Stoloff SW, Stempel DA, Meyer J, et al. Improved refill persistence with fluticasone propionate and salmeterol in a single inhaler compared with other controller therapies. J Allergy Clin Immunol 2004; 113: 245-251.

14 Marceau C, Lemière C, Berbiche $\mathrm{D}$, et al. Persistence, adherence, and effectiveness of combination therapy among adult patients with asthma. J Allergy Clin Immunol 2006; 118: 574-581.

15 Cuerq A, Pépin S, Ricordeau P. Remboursement de médicaments antiasthmatiques: une approche de la prévalence et du contrôle de l'asthme. [Reimbursement of anti-asthma therapy: an approach to asthma prevalence and level of disease control.] Points de Repère, numéro 24, December 2008.

16 Navaratnam P, Jayawant SS, Pedersen CA, et al. Physician adherence to the national asthma prescribing guidelines: evidence from national outpatient survey data in the United States. Ann Allergy Asthma Immunol 2008; 100: 216-221.

17 Williams LK, Peterson EL, Wells K, et al. Quantifying the proportion of severe asthma exacerbations attributable to inhaled corticosteroid nonadherence. J Allergy Clin Immunol 2011; 128: 1185-1191. 